\title{
Implantação de mentoria on-line em uma faculdade de medicina durante a pandemia da Covid-19
}

\author{
Implementation of online mentoring at a medical school during the Covid-19 pandemic
}

\author{
Sandra Torres Serra' (D) sandratserra@gmail.com \\ Mariana Bteshe ${ }^{1}$ (D) marianabteshe@gmail.com \\ Ricardo Bedirian'1 (D) rbedirian@gmail.com \\ Daniela Santos Belz ${ }^{1}$ (D) santosdanielabelz@gmail.com \\ Carolina Figueira Franco ${ }^{1}$ (D) cfigueirafranco@gmail.com

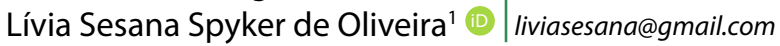

\begin{abstract}
RESUMO
Introdução: Com a interrupção das aulas presenciais nas escolas médicas ocasionada pela pandemia da doença pelo novo coronavírus, verificouse que os alunos ingressantes no primeiro ano de Medicina de uma faculdade pública brasileira apresentavam maior vulnerabilidade social e psicológica devido aos vínculos pouco consolidados com colegas e com o ambiente acadêmico. Observou-se que os fatores de estresse relacionados às adaptações ao contexto universitário se intensificaram, tornando essa turma prioritária para o desenvolvimento de intervenções emergenciais de suporte e acolhimento. Este trabalho relata um projeto-piloto de mentoria entre professores e alunos em ambiente virtual, visando à sua posterior institucionalização como atividade regular. Descreve etapas de implantação, desenvolvimento, resultados iniciais e perspectivas.
\end{abstract}

Relato de experiência: Foi desenvolvida uma mentoria on-line entre alunos e professores, em que utilizou a dinâmica de grupos de reflexão como estratégia metodológica. Por meio de redes sociais e reuniões on-line, alunos e professores foram convidados a participar do projeto, que contou com seis docentes, um preceptor e 29 alunos, além da equipe coordenadora. Realizou-se uma capacitação on-line de professores com conteúdos teóricos e simulações, objetivando delimitar o papel deles, os objetivos, o enquadre e as especificidades de uma mentoria remota. A avaliação final da atividade foi feita por professores e alunos.

Discussão: As medidas de distanciamento social evidenciaram a necessidade de oferecer oportunidades de aprendizagem e de convívio mediadas por plataformas digitais. Comprovou-se a adesão de alunos e professores à mentoria na modalidade on-line, e observou-se a sua potencialidade para fomentar uma relação aluno-professor baseada na troca de ideias, reflexão e suporte social. Constatou-se também um incremento da motivação para a adesão ao curso.

Conclusão: A mentoria on-line proporcionou benefícios aos participantes, corroborando resultados de experiências no modelo tradicional presencial, e instigou a realização de pesquisas que aprofundem a investigação sobre a sua eficácia e relevância para a formação médica.

Palavras-chave: Educação Médica; Tutoria; Estudantes de Medicina; Epidemia pelo Novo Coronavírus.

\begin{abstract}
Introduction: With the interruption offace-to-face classes in medical schools caused by the Covid-19 pandemic, new first-year medical students at a Brazilian public university showed greater social and psychologic vulnerability due to poorly consolidated ties with colleagues and the academic environment. Intensified stress factors related to adapting to the university environment have been observed, making this group a priority for emergency support and reception interventions. This article reports on a pilot online mentoring project, between teachers and students in a virtual environment, with a view to its subsequent institutionalization as a regular activity. The stages of implementation, development, initial results, and perspectives are described.
\end{abstract}

Experience report: The pilot project aimed to operationalize online mentoring between teachers and students, using reflection group dynamics as a methodological strategy. Through social networks and online meetings, students and teachers were invited to participate in the project, which included six teachers, one preceptor, and twenty-nine students, in addition to the coordination team. Online teacher training with theoretical content and simulations was conducted to outline the teacher's role in the activity, task objectives, framework, and specific characteristics of online mentoring. The final evaluation was performed by teachers and students.

Discussion: Social distancing measures have evinced the need for online learning and socializing opportunities. Gathering students and teachers in an online mentoring activity has proven to be feasible and its potential to foster a student-teacher relationship based on exchange of ideas, reflection and social support was observed. Increased motivation to join the course was observed.

Conclusion: Online mentoring has brought benefits to the participants, corroborating the results of experiences in the traditional face-to-face model, and has instigated research that deepens investigation into its effectiveness and relevance for medical training.

Keywords: Medical Education; Mentoring; Medical Students; Covid-19 Pandemic.

${ }^{1}$ Universidade do Estado do Rio de Janeiro, Rio de Janeiro, Rio de Janeiro, Brasil.

Editora: Lia Marcia Cruz da Silveira.

Recebido em 25/02/21; Aceito em 04/05/21.

Avaliado pelo processo de double blind review. 


\section{INTRODUÇÃO}

A construção de vínculos em um contexto pandêmico que impôs medidas de isolamento e distanciamento social foi um dos grandes desafios enfrentados por alunos e professores nas escolas médicas durante a pandemia da coronavirus disease 2019 (Covid-19). O distanciamento físico necessário para frear o contágio ocasionou uma série de paralisações nas atividades presenciais curriculares e extensionistas. Em resposta à necessidade de continuidade da formação, direcionou-se a aprendizagem para espaços virtuais, e intervenções pedagógicas e de suporte ao aluno mediadas por tecnologias de informação e comunicação foram aplicadas nas escolas médicas ${ }^{1}$.

Na perspectiva de suporte psicológico, desde o início da decretação das medidas de distanciamento social, o Programa de Apoio Psicopedagógico ao Estudante (Pape) da Faculdade de Ciências Médicas (FCM) da Universidade do Estado do Rio de Janeiro (Uerj) manteve atendimento clínico individual aos alunos, realizado de forma virtual por meio de chamadas de vídeo ou de áudio, e, durante três meses consecutivos, promoveu rodas de conversa para a comunidade interna por meio de plataforma virtual, em que se debateram os temas emergentes relacionados à pandemia. A manutenção dos atendimentos individuais e a oferta de espaços dialógicos grupais em modalidade virtual foram medidas positivas para o acolhimento e suporte aos alunos diante da realidade que se apresentava, porém observávamos que os sintomas de ansiedade, depressão e desamparo permaneciam prevalentes.

A observação empírica foi corroborada por um levantamento realizado pelo Centro Acadêmico Sir Alexander Fleming (Casaf) respondido por 609 alunos que compõem o corpo discente da faculdade. Destes respondentes, $86,4 \%$ relataram algum tipo de impacto sofrido em sua saúde mental decorrente da pandemia, sendo a depressão e ansiedade os quadros mais citados. Evidenciou-se que os alunos ingressantes no primeiro ano apresentavam maior vulnerabilidade social devido aos vínculos pouco consolidados com os colegas de turma e com o próprio ambiente acadêmico, visto que as aulas foram interrompidas com apenas uma semana de atividades presenciais, em março de 2020. Além disso, observouse que fatores de estresse relacionados às adaptações ao contexto universitário, comumente identificados em alunos ingressantes ${ }^{2-5}$, intensificaram-se, tornando essa turma prioritária para o desenvolvimento de intervenções emergenciais de suporte e acolhimento.

Contudo, observávamos que os professores também enfrentavam inúmeros desafios. Não somente foram exigidos a se adaptar rapidamente ao ensino remoto, como a crise que incidiu sobre a educação médica suscitou questionamentos sobre o papel do professor no novo contexto. Diante de tantas desestabilizações no processo de ensino-aprendizagem, os professores também se ressentiam do afastamento de alunos com as medidas de isolamento.

A realização de uma mentoria mediada por plataforma digital nos pareceu possuir uma viabilidade operacional compatível com a urgência do momento, apresentando possibilidades de aproximação imediata das pessoas e permitindo que alunos e professores pudessem se conectar de novas maneiras. Optamos por um programa em modalidade de mentoria grupal, constituindo grupos de alunos facilitados por um mentor, com o objetivo de que eles compartilhassem sentimentos e ideias e refletissem juntos sobre os impactos na formação e as possíveis soluções para os impasses acadêmicos e pessoais advindos com a experiência do distanciamento social.

\section{Opção metodológica para a mentoria on-line e a nossa experiência anterior}

O funcionamento de um projeto-piloto de mentoria on-line segundo a dinâmica de funcionamento de um grupo de reflexão foi considerada a escolha mais adequada para atender aos nossos objetivos e ao senso de urgência imposto pela pandemia. $\mathrm{O}$ grupo de reflexão é reconhecido pelo seu papel estratégico de acolhimento das angústias e crises dos estudantes durante a formação médica. Entre suas funções, destacam-se: fomentar o diálogo, facilitar o vínculo entre estudantes, professores e a instituição, e promover a convivência colaborativa. A troca de vivências durante a graduação possibilita que os membros do grupo possam escutar as dificuldades enfrentadas pelo outro, as estratégias para lidar com os desafios e as diferentes emoções que dali surgem. Por meio da identificação, do espelhamento e das ressonâncias grupais, os participantes podem construir um lugar de vivências, divergências e contradições capaz de dar um novo sentido às suas histórias, favorecer a criação de vínculos positivos e permitir que juntos busquem soluções para o grupo ${ }^{6-8}$.

Após a escolha metodológica, na organização do projeto-piloto resgatamos os resultados positivos, os desafios e os motivos da interrupção do "Projeto Tutoria (Mentoring)", desenvolvido na instituição entre 2002 e 2012. Enquanto esteve em funcionamento, essa experiência obteve benefícios assemelhados aos programas de mentoria ${ }^{9}$ relatados na literatura: incremento da relação aluno-professor, maior conscientização pelas escolhas de carreira, aumento de produtividade em projetos de pesquisa, melhor desempenho acadêmico e aumento de percepção de bem-estar. Na FCM da Uerj constituiu-se como um espaço de reflexão sobre a vida acadêmica, objetivando principalmente as resoluções das tensões ligadas aos estudos, à formação e ao trabalho prático. Além disso, esse projeto possibilitou aos alunos a elaboração dos conflitos experimentados nas relações interpessoais, no âmbito 
da convivência institucional ${ }^{10}$. Os fatores que influenciaram a extinção dessa atividade, tais como as questões acerca de adesão de alunos e professores, horário e periodicidade de reuniões e temário de discussões, também foram considerados e amplamente discutidos entre a equipe coordenadora e os professores participantes para a reorientação da implantação da mentoria na modalidade on-line.

Nosso desafio a partir de então consistiu na redefinição de objetivos e enquadres para a adequação de implantação do modelo ao formato digital. Consideramos que o exercício da mentoria on-line como projeto-piloto poderia oferecer subsídios tanto para impulsionar uma elaboração adequada às circunstâncias e necessidades atuais quanto para fundamentar os argumentos para uma futura institucionalização.

\section{RELATO DE EXPERIÊNCIA}

\section{Implementação: proposta e construção pactuada}

A atividade foi desenvolvida em uma faculdade pública de Medicina como projeto-piloto intitulado"Grupos de Reflexão online para Estudantes e Professores" no período compreendido entre os meses de outubro e dezembro de 2020. A proposta de funcionamento foi operacionalizar uma mentoria em grupo mediada por plataforma digital, sendo o mentor um professor, e os mentorados um grupo de alunos do primeiro ano.

Alunos foram convidados por intermédio de representantes do centro acadêmico para compor a equipe responsável pela implantação da atividade. Em seguida, marcou-se uma reunião para apresentação da proposta com três alunas da turma de calouros interessadas em participar da organização. Nesse esse primeiro diálogo, muitos pontos foram abordados, como o levantamento realizado pelo centro acadêmico que mostrou a urgência no cuidado com a saúde mental dos estudantes e a importância de esse projeto oferecer apoio emocional para os alunos se adaptarem à implantação do ensino remoto, a dificuldade de acesso digital e as limitações de distância física. Foi ressaltada a demanda da turma de ingressantes para criação de canais de comunicação com professores e demais instâncias da faculdade - visto que ainda não haviam estabelecido laços com a universidade. Em seguimento, realizou-se uma série de reuniões com o grupo de alunas para determinar como o projeto seria implementado de forma prática na instituição. Elaborou-se um planejamento de divulgação do projeto e de seus objetivos por meio de redes sociais, de forma a sensibilizar os alunos da turma a participar. Em um segundo momento, foi disponibilizado um link para inscrição via formulário eletrônico, no qual os alunos interessados deveriam expor suas expectativas sobre o projeto e escolher os horários disponíveis para participar das reuniões, bem como elencar os assuntos que esperavam abordar.
Em paralelo, a coordenação de graduação discutia com os professores os desafios impostos pela pandemia, promovendo rodas de conversas sobre a temática "Desafios da Docência em Tempos de Pandemia". Realizaram-se quatro encontros por videoconferência com a participação de cerca de 20 professores em cada. A proposta de realização da atividade de grupos de reflexão para alunos e professores foi apresentada nesse fórum e dirigiu-se um convite ao grupo de docentes, quando emergiram os primeiros voluntários para a atividade. O convite foi posteriormente estendido a todo corpo docente e preceptores, resultando na adesão de seis professores e um preceptor.

\section{Enquadre da atividade e desenvolvimento}

Definido o grupo de professores participantes, demos seguimento às reuniões de planejamento metodológico. Em um movimento de construção partilhada e colaborativa, a equipe coordenadora, os professores e as alunas acordaram o enquadre da atividade estruturado e posteriormente desenvolvido sob os seguintes fundamentos:

- Objetivos:

1) Ofertar um espaço seguro no qual estudantes e professores pudessem trocar experiências, dificuldades, desafios e soluções para a aprendizagem e convivência em tempos de distanciamento social.

2) Consolidar uma rede de suporte e de pertencimento entre professores e alunos, por meio da promoção de encontros virtuais.

3) Favorecer a criação de vínculos de confiança e colaborativos entre alunos e entre alunos e professores.

- Modalidade: mentoria on-line em grupo, reunindo um professor-mentor e um grupo de até dez alunos do primeiro ano do curso médico.

- Espaço: encontros on-line por videoconferência, mediados pela plataforma digital oficial da instituição. As reuniões não foram gravadas para garantir a privacidade e o sigilo do grupo.

- Público-alvo: alunos ingressantes no primeiro ano do curso em 2020, com participação facultativa. Por meio de formulário on-line, 29 alunos (21 do sexo feminino e oito do sexo masculino) se inscreveram. Todos os inscritos participaram da atividade.

- Composição dos grupos: os alunos foram distribuídos nos grupos segundo seus horários de disponibilidade.

- Mentores: seis professores, sendo um de Patologia Geral, um de Farmacologia, um de Pneumologia, 
três de Medicina Integral Familiar e Comunitária e um preceptor de Hematologia, totalizando sete mentores, todos com participação voluntária.

- Periodicidade: mínimo de quatro e máximo de seis encontros dos mentores com seu respectivo grupo, durante o período de outubro a dezembro de 2020, de cerca de uma hora e meia. Além das reuniões online, os participantes dos grupos se comunicaram por meio de aplicativos para telefones celulares.

- Temário: foi acordado que o próprio grupo decidiria sobre as temáticas a serem discutidas, exceto na primeira reunião, na qual se realizaram o enquadre da atividade, o esclarecimento dos objetivos, a apresentação dos componentes, as definições de regras de convivência e o compartilhamento de expectativas e relatos sobre a situação de isolamento social.

- Seleção e treinamento de professores: todos os professores interessados em participar foram contemplados. Realizou-se uma capacitação de professores antes das reuniões dos grupos.

- Supervisão de professores: além das reuniões de organização preparatórias, houve quatro reuniões de acompanhamento e supervisão com a coordenação do projeto.

- Avaliação da atividade: feita por professores e alunos na última reunião de grupo, por meio de feedback dos discentes em forma de narrativas orais que avaliaram os impactos e desdobramentos da atividade na formação pessoal e acadêmica.

- Coordenação do projeto: realizada pela equipe do Pape e pela coordenação de graduação da FCM.

- Inserção institucional: o projeto foi cadastrado como projeto de extensão no Departamento de Extensão da universidade. Emissão de certificado de participação para os alunos mediante $75 \%$ de frequência.

\section{Capacitação e supervisão de professores}

Foi promovida e realizada pelo Pape uma capacitação on-line que objetivou potencializar o conhecimento dos professores sobre o trabalho em pequenos grupos, discutindo os problemas inerentes ao ensino de graduação e as questões relacionadas ao distanciamento social. Teve uma carga horária de quatro horas, divididas em duas reuniões de cerca de duas horas cada, quando se reforçaram os objetivos e as especificidades da tarefa, e delimitou-se o papel do professor na atividade. $O$ tempo das reuniões de capacitação foi dividido em uma parte teórica e uma parte prática. Na parte teórica, estudamos as diferentes funções que um profissional mais experiente pode assumir ao acompanhar o aluno na formação ${ }^{11}$. Foi pactuado que o professor assumiria o papel de mentor para um grupo de alunos, a fim de fomentar o diálogo, o vínculo e a partilha de ideias, afetos e sentimentos de forma reflexiva, e oferecer-lhes acolhimento e suporte para o desenvolvimento acadêmico e pessoal. Na capacitação também foram discutidas com os professores as dificuldades de adesão de alunos à atividade, observadas na nossa experiência anterior e em outras escolas ${ }^{12}$.

Na parte prática, realizaram-se simulações on-line de reuniões com alunos seguidas de debate, com o objetivo de introduzir os professores de forma dinâmica na tarefa que viriam a desempenhar.

Em quatro reuniões de supervisão on-line, os professores relataram o desenvolvimento das reuniões realizadas e as interações estabelecidas entre os participantes, destacando as principais questões debatidas e seus encaminhamentos. Além do horário agendado, criou-se um grupo por aplicativo de celular para facilitar a comunicação, constituindo-se como mais um espaço de troca e suporte para as ações dos professores.

\section{DISCUSSÃO}

Durante a paralisação de atividades presenciais e as medidas de distanciamento social, o uso de plataformas digitais se apresentou como uma alternativa ao convívio social e ao estabelecimento de vínculos de pertencimento.

Cabe ressaltar o caráter inovador na estruturação e condução desse projeto, fruto de uma colaboração inédita entre diversos setores da faculdade - coordenação de graduação, programa de apoio psicopedagógico, centro acadêmico e corpo discente. O protagonismo de seus participantes fez com que o projeto implementado fosse, desde a sua criação, uma construção coletiva e pensada na pluralidade do ambiente acadêmico. As parcerias estabelecidas entre alunos e professores no planejamento e na implementação do projeto contribuíram para o êxito da atividade, uma vez que obtivemos uma adesão inicial de $20 \%$ dos alunos da turma, considerada satisfatória se comparada a trabalhos publicados ${ }^{12}$ e à nossa própria experiência anterior. Outro fator que pode ter contribuído para a adesão de alunos foi a oferta de horários para a realização dos encontros (em períodos livres de atividades na grade curricular), mais ampla e flexível do que aqueles oferecidos em mentorias presenciais.

O enfrentamento da pandemia no cotidiano familiar e na vida pessoal, e a rotina adaptada para o estudo remoto foram temas predominantes nas reuniões. Os alunos tiveram a oportunidade de expressar as suas dificuldades de ordem acadêmica e emocional, que foram acolhidas e trabalhadas, na medida do possível, no espaço grupal. 
No feedback dado pelos alunos a respeito do impacto da atividade, eles revelaram que se sentiram acolhidos pelos professores e colegas do grupo, e ainda ampliaram suas redes de conexões a outros colegas da turma. Observou-se a construção de um senso de pertencimento à faculdade, apesar de um contexto extremamente adverso e atípico, onde conviveram presencialmente por poucos dias apenas. Professores e alunos tiveram a oportunidade de compartilhar seus sentimentos e suas percepções sobre os primeiros meses de ensino remoto, e expressar espontaneamente, em um debate plural, as soluções que encontraram para lidar com as dificuldades enfrentadas decorrentes da crise sanitária. Os alunos queixaram-se da falta de tempo para o cumprimento das demandas acadêmicas e expressaram sentimentos de ansiedade associados. Conversar com colegas sobre situações, angústias e problemáticas similares, tanto quanto com professores - que apresentaram visões diferenciadas das questões debatidas -, ajudou os alunos a construir vínculos e um sentimento de identidade grupal.

Os professores relataram que puderam conhecer mais proximamente os alunos participantes: a situação atual de moradia, de estudo, acessibilidade a meios digitais para o ensino remoto, composição e ambiente familiar, pontos relevantes da história pessoal e expectativas em relação ao curso médico.

Em termos pedagógicos, obteve-se uma amostra das dificuldades e dos pontos de conflito que estão em pauta no primeiro ano do curso médico, suscitando entre o grupo de professores-mentores algumas reflexões sobre como encaminhar soluções para questões pedagógicas, éticas ou relacionais que envolvessem outras disciplinas e colegas.

Assuntos relacionados ao ensino na modalidade remota foram abordados nas reuniões, tais como o tempo excessivo de aulas síncronas, as cobranças de realização de atividades extras e as avaliações não pactuadas entre determinadas disciplinas e a turma. Algumas questões específicas acerca da realização dos grupos de mentoria em ambiente virtual foram discutidas durante as supervisões com os professores, como sigilo das reuniões e os limites da privacidade no ambiente familiar e social no momento da realização da atividade.

O programa de capacitação de professores compreendendo a discussão de textos científicos e de situações simuladas foi uma metodologia exitosa para esclarecer qual o papel do professor nos grupos. Delinear as diferentes funções que o professor pode assumir no âmbito acadêmico foi um passo inicial importante para pactuar entre os professores que sua função nos grupos seria a de um mentor. Nessa função, a relação com o seu grupo de mentorados se estrutura como uma relação formativa e ao mesmo tempo confidencial, favorável ao desenvolvimento de laços de confiança ${ }^{13}$, que permitam "conjugar ao mesmo tempo o suporte pessoal e o desenvolvimento profissional" (p. 55) ${ }^{14}$.

Comoresultado complementare não esperado, a atividade mostrou ser um espaço fértil para o desenvolvimento docente, pois, além de possibilitar aos professores travar conhecimento com colegas de outras áreas, permitiu a troca de experiências pedagógicas exitosas, ao informarem-se sobre atividades de extensão ou propostas inovadoras de metodologias de ensino e avaliação de suas respectivas disciplinas.

Em uma avaliação preliminar, verificamos resultados positivos do projeto-piloto de mentoria on-line e a sua potencialidade para se consolidar como uma atividade institucional regular. Em um contexto pandêmico e de distanciamento social, a mentoria na modalidade on-line ampliou a rede de suporte psicológico e social tanto para alunos quanto para professores, e, por meio de uma metodologia reflexiva, crítica e ativa, estabeleceu um espaço seguro para partilha de expectativas, angústias e preocupações sobre a formação acadêmica.

\section{CONSIDERAÇÕES FINAIS}

A crise sanitária e as medidas de distanciamento social evidenciaram a necessidade de incentivar e oferecer oportunidades de aprendizagem e de convívio on-line. As possibilidades de utilização do ambiente virtual para fins de entrosamento social e articulada a propósitos pedagógicos constitui um tema de investigação necessário nos dias atuais. Nesse projeto-piloto de funcionamento de grupos em conformidade à modalidade de mentoria on-line, foi verificada a sua potencialidade em estimular o contato entre alunos e professores, fomentando uma relação mútua de troca de ideias e suporte social. Os resultados obtidos superaram as expectativas, ensejando aos participantes - professores e alunos - a sua continuidade no âmbito institucional, estando em vias de organização a realização de um novo ciclo de reuniões grupais. A mentoria on-line não só trouxe benefícios aos participantes, corroborando resultados de experiências exitosas do modelo tradicional presencial, como instigou a realização de pesquisas que aprofundem a investigação sobre a eficácia ou relevância da mentoria mediada por tecnologia digital. Identificar os fatores de interesse e também os fatores de recusa à participação na atividade é fundamental para a sua formalização institucional e para o desenvolvimento de estratégias que favoreçam a adesão de alunos e professores à iniciativa.

Uma vez implantada institucionalmente, uma avaliação sistemática permitirá elaborar estratégias de reformulação para adequação às necessidades de alunos e professores, de forma que o projeto possa ser estendido e oferecido aos outros períodos do curso de Medicina e permanecer em funcionamento mesmo após o período de distanciamento social. 


\section{CONTRIBUIÇÃO DOS AUTORES}

Todos os autores participaram ativamente de todas as etapas de elaboração e da aprovação final do manuscrito.

\section{CONFLITO DE INTERESSES}

Declaramos não haver conflito de interesses.

\section{FINANCIAMENTO}

Declaramos não houver financiamento.

\section{REFERÊNCIAS}

1. Gordon M, Patricio M, Horne L, Muston A, Alston SR, Pammi M, et al. Developments in medical education in response to the COVID-19 pandemic: a rapid BEME systematic review: BEME Guide No.63. Med Teach. 2020;42(11):1-14.

2. Bellodi, PL. O Programa Tutores e a integração dos calouros na FMUSP. Rev Bras Educ Med. 2004;28(3):204-14. doi: 10.1590/1981-5271v28.3-027.

3. Cameron D, Dromerick LJ, Ahn J, Dromerick AW. Executive/life coaching for first year medical students: a prospective study. BMC Med Educ. 2019;19(1):163. doi:10.1186/s12909-019-1564-4.

4. Melo Filho J. A vocação médica e o curso médico. In: Melo Filho J, organizador. Identidade médica: implicações históricas e antropológicas. São Paulo: Casa do Psicólogo; 2006. p. 59-159.
5. Millan LR, Rossi E, De Marco OLN. A psicopatologia do estudante de medicina. In: Millan LR, De Marco OLN, Rossi E, de Arruda PCV organizadores. Universo psicológico do futuro médico: vocação, vicissitudes e perspectivas. São Paulo: Casa do Psicólogo; 1999. p. 83-94.

6. Fernandes BS. Como trabalho com grupo de reflexão. Rev. SPAGESP. 2000;1(1):77-82.

7. Guanaes C, Mattos ATR. O grupo de reflexão na formação do profissional de saúde: um enfoque construcionista social. Gerais (Univ Fed Juiz de Fora). 2008;1(1):79-85.

8. Muniz JR, Chazan LF. Ensino de psicologia médica. In: Mello Filho J, organizador. Psicossomática hoje. Porto Alegre: Artmed; 1992. p. 37-44.

9. Frei E, Stamm M, Buddeberg-Fischer B. Mentoring programs for medical students: a review of the PubMed literature 2000-2008. BMC Med Educ. 2010;10(1):32-56. doi:10.1186/1472-6920-10-32.

10. Serra ST.Tutoria e a possibilidade de construção da identidade profissional. In: Melo Filho J, organizador. Identidade médica: implicações históricas e antropológicas. São Paulo: Casa do Psicólogo; 2006. p.191-211.

11. Botti SHO, Rego S. Preceptor, supervisor, tutor e mentor: quais são seus papéis? Rev Bras Educ Med. 2008;32(3):363-73. doi: 10.1590/S010055022008000300011.

12. Bellodi PL, Chebabo R, Abensur SI, Martins MA. Mentoring: ir ou não ir, eis a questão: um estudo qualitativo. Rev Bras Educ Med. 2011;35(2):237-45. doi: 10.1590/S0100-55022011000200013.

13. Walsh K. Online mentoring in medical education. South African Family Practice. 2016;58(1):7-8. doi: 10.1080/20786190.2015.1059020.

14. Bellodi PL, Martins M. Tutoria: mentoring na formação médica. São Paulo: Casa do Psicólogo; 2005. 